\title{
The Meyer- Neldel Rule in $\mathrm{Se}_{70} \mathrm{Te}_{30-\mathrm{x}} \mathrm{In}_{\mathrm{x}}$
}

\author{
A. Abd-El Mongy \\ Department of Physics, Faculty of Science, Helwan University, \\ Ein Helwan, Cairo, Egypt.
}

The electrical conductivity in $\mathrm{Se}_{70} \mathrm{Te}_{30-x} \operatorname{In}_{x}(0<x<10)$ at. \% exhibited an exponential thermally activated behavior with single conduction mechanism for bulk and thin films. Film conductivities were found to be about three orders of magnitude lower than those of their bulk counterparts bulk samples. An obvious decrease in the conductivity on incorporation of indium to the binary $\mathrm{Se}_{70} \mathrm{Te}_{30-x}$ film was observed. An experimental correlation between the activation energy and the pre-exponential factor was observed indicating the validity of Meyer-Neldel rule in the studied samples. Huge difference in $\sigma_{0}$ values was obtained and hence $\sigma_{0}$ may not directly related to the microscopic conductivity. 


\section{Introduction:}

In semiconducting materials the dc conductivity $(\sigma)$ near room temperature is found to obey Armenia's formula:

$$
\sigma=\sigma_{\mathrm{o}} \exp (-\Delta \mathrm{E} / \mathrm{KT})
$$

Surprisingly, there is a quite often an exponential relation between the activation energy $\Delta \mathrm{E}$ and the pre-exponential factor $\sigma_{0}$, known as the MeyerNeldel (MN) rule [1]. The MN rule can be written as:

$$
\sigma_{\mathrm{o}}=\sigma_{\mathrm{oo}} \exp (\Delta \mathrm{E} / \mathrm{KTMN})
$$

where $\sigma_{\mathrm{oo}}$ is the pre-pre-exponential and $\mathrm{T}_{\mathrm{MN}}$ is a characteristic temperature. The relation has been observed experimentally in a wide variety of single crystal and polycrystalline semiconductors [2], in hydrogenated amorphous silicon [3\&4], liquid semiconductor [5], and in binary alloys [6]. Although several models have been proposed to explain the MN rule, each explanation is valid only for limited class of solids. In organic semiconductor a model of thermally excited electron tunneling through intermolecular barriers is suggested [7]. In amorphous hydrogenated silicon, Irsingler et al. [8] suggested that whenever the conductivity activation energy is changed the conductivity pre-exponential factor has to be corrected accordingly. In liquid semiconductor, Fortner et al. [5] assumed that multiphonon hopping could explain MN rule of the electrical conductivity. Narasimhan and Arora [6] showed the validity of $\mathrm{MN}$ rule in III-V compound semiconductor and reported $\mathrm{E}_{\mathrm{MN}}\left[\Delta \mathrm{E} / \mathrm{KT}_{\mathrm{MN}}\right]$ of $(25-50 \mathrm{meV})$. Coutts and Pearsall [2] reported $\mathrm{E}_{\mathrm{MN}}=40 \mathrm{meV}$ for some chalcogenides. Dwivedi [9] reported $\mathrm{E}_{\mathrm{MN}}(0.2-0.7 \mathrm{eV})$ for some amorphous chalcogenide systems. Roberts [10] assumed that if the tail states are exponential with energy and Fermi level is controlled by deeper level in the gap, the MN rule is obeyed. Jackson [11] concluded that the energy factor of the $\mathrm{MN}$ rule is related to the energy distribution of the traps while the pre-factor is related to the microscopic transport properties.

Evidence for the validity of the MN rule in chalcogenides has not been frequently reported although sufficient data on conductivity are available in the literature. The object of the present work was then to investigate the dc conductivity of the system $\mathrm{Se}_{70} \mathrm{Te}_{30-\mathrm{x}} \operatorname{In}_{\mathrm{x}}(0<\mathrm{x}<10)$ around room temperature. Study was also focused on comparing the conductivity parameters in bulk and film samples. In addition it was aimed to examine whether or not the MN rule is obeyed in such chalcogenides. Theoretical models, proposed for the MN rule, are presented and the results are analyzed with the help of these models. 


\section{Experimental procedure:}

Glassy alloys of the system $\mathrm{Se}_{70} \mathrm{Te}_{30-\mathrm{x}} \operatorname{In}_{\mathrm{x}}(\mathrm{x}=0,2.5,5,7.5,10)$ were prepared by the normal quenching technique. The raw materials $(99.999 \%$ pure) were weighted according to their atomic percentages and sealed in an evacuated $\left(10^{-5}\right.$ Torr) quartz ampoule. The ampoules were heated at a temperature around $850{ }^{\circ} \mathrm{C}$ for 30 hours, under air atmosphere with continuous rotating (using a mechanical system) to make homogeneous melt. The melt was quenched in ice - water and cast into a disc of $30 \mathrm{~mm}$ in diameter and $2 \mathrm{~mm}$ thick. Careful grinding and polishing were done before preserving samples in a desicator.

Films were made from the as- prepared alloys by using a vacuum evaporation technique (Edwards coating system E306A) at room temperature and pressure of $10^{-5}$ Torr. During the film preparation, the thickness was controlled by means of a thickness monitor. After deposition an optical method, by applying multiple-beam Fizeau fringes at reflection, was used to give the accurate film thickness (about 70nm).

The potential difference between hot and cold contacts was used to test the conductivity type. The planar geometry configuration was used for the conductivity measurement. The electrode geometry was measured using a travelling microscope. Aluminum electrodes were deposited at vacuum of about $10^{-3}$ Torr and a thin layer of silver paste was used to ensure good ohmic contacts. The ohmic nature of the contacts was investigated by applying the voltage in both directions and measuring the corresponding current. A constant voltage (in the ohmic region) was applied and a Keithley electrometer was used to detect the current passing through the samples. The temperature was measured in the range $300 \mathrm{~K}<\mathrm{T}<360 \mathrm{~K}$ by means of an Oxford temperature controller (DTC2). Using the dimensions of the specimens, the distance between electrodes $\mathrm{L}$, the electrode width $\mathrm{b}$, the dc conductivity was calculated according to the relation $\sigma=1 /(\mathrm{Rdb})$, where $\mathrm{R}$ and $\mathrm{d}$ are the resistance and the thickness of the sample respectively.

\section{Results:}

The amorphous nature of both bulk and film samples was proved by $\mathrm{XRD}$, using Diano diffractometer ( $\mathrm{Ni}$ filtered $\mathrm{Co}-\mathrm{K} \alpha$ radiation at $50 \mathrm{kV}$ and $30 \mathrm{~mA})$.

The hot-point probe method showed that the investigated samples are p-type. In fact a weak response was observed for film specimens, which was attributed to their high resistivities. 
Figures $(1 \&$ \& $)$ show the temperature dependence of the $\mathrm{dc}$ conductivity in bulk and film samples respectively for different indium concentration. For all samples and in the measured temperature range the plot of $\ln \sigma-1 / \mathrm{T}$ showed an activated behaviour with one mode of conduction. The straight lines indicated the validity of Arrhenius equation. From the slope and the intercept (extrapolation of the line to $1 / \mathrm{T}=0$ ), the activation energy $\Delta \mathrm{E}$ and the conductivity pre-exponential $\sigma_{o}$ values are calculated subsequently. Inspection of results reveals the increase of conductivity (decrease of $\Delta \mathrm{E}$ ) as indium content is increased in bulk and film forms. However in film samples the conductivity of the specimen without indium showed anomalous behavior. Figures $(3 \& 4)$ depict a linear dependence of $\ln \sigma_{0}$ when plotted versus $\Delta \mathrm{E}$. The slope of these lines yield $\left(\mathrm{KT}_{\mathrm{MN}}\right)^{-1}=21.36(\mathrm{eV})^{-1}$ and intercept at $\sigma_{00}$ (line extrapolated to $\Delta \mathrm{E}=0)=3.75 \times 10^{-3} \mathrm{~S} \mathrm{~cm}^{-1}$ in bulk while in film specimens the respective values are $26.76(\mathrm{eV})^{-1} \& 2.9 \times 10^{-6} \mathrm{~S} \mathrm{~cm}^{-1}$. As can be seen the magnitude of $\sigma_{0}$ in film samples of different In concentration is very much different.

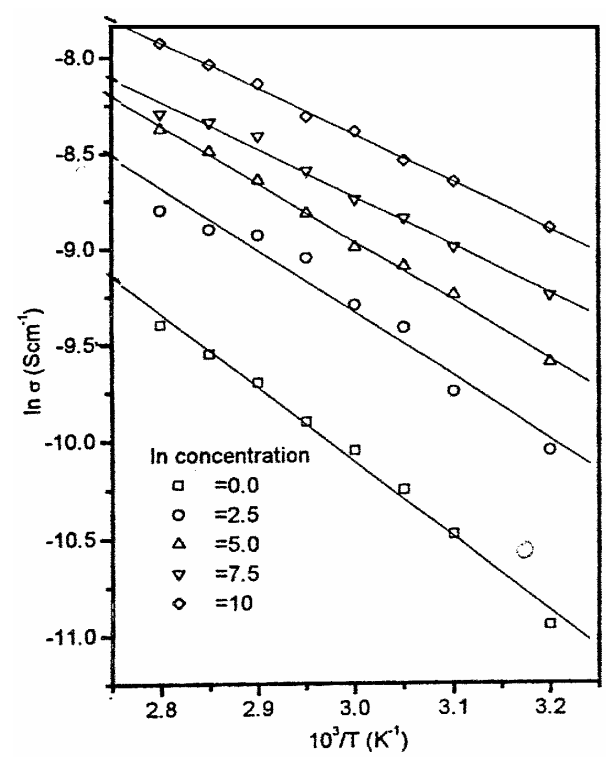

Fig. (1): The plot of $\ln \sigma$ and $1 / \mathrm{T}$ for bulk samples at different $\ln$ concentration.

Fig. (2): $\ln \sigma-1 / \mathrm{T}$ plot for thin film specimens at different $\ln$ concentration. 


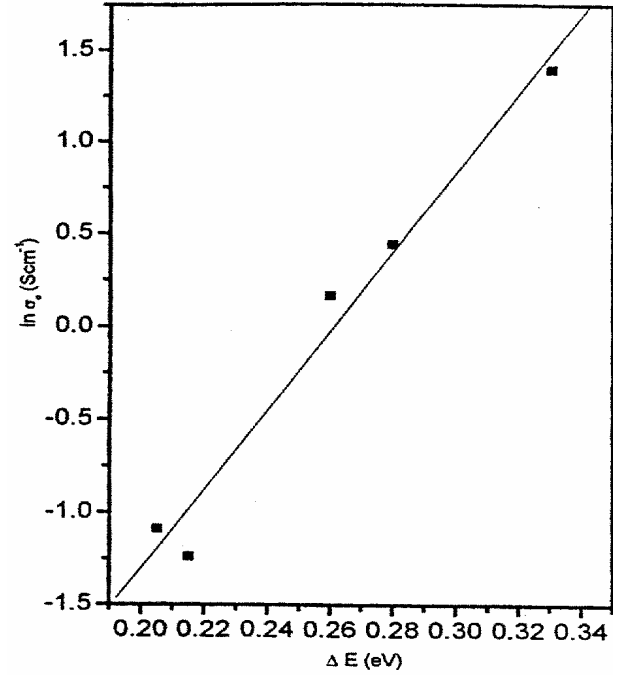

Fig. (3): The plot of in $\sigma_{\mathrm{o}}$ versus activation energy for bulk samples.

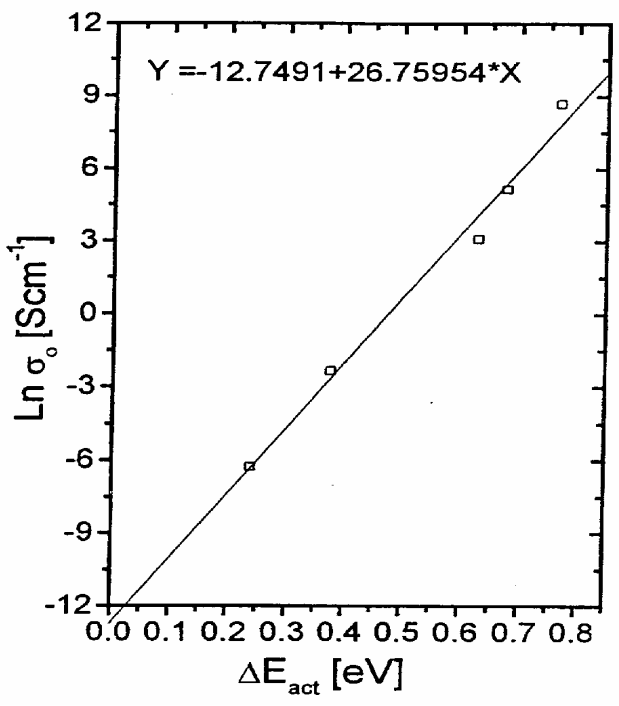

Fig. (4): $\ln \sigma_{o}-\Delta E$ plot for thin films.

Table (1): Conducting parameters for the system $\mathrm{Se}_{70} \mathrm{Te}_{30-\mathrm{x}} \mathrm{In}_{\mathrm{x}}$, bulk samples: $\sigma_{00}=3.75 \times 10^{-3} \mathrm{~S} \mathrm{~cm}^{-1},\left(\mathrm{KT}_{\mathrm{MN}}\right)^{-1}=21.36(\mathrm{e} \mathrm{V})^{-1}$

\begin{tabular}{|c|c|c|}
\hline In Con. $(\boldsymbol{a t} \%)$ & $\Delta \boldsymbol{E}(\boldsymbol{e} \boldsymbol{V}) \pm \mathbf{0 . 0 1}$ & $\boldsymbol{\sigma}_{\boldsymbol{o}}\left(\mathbf{S ~ c m}^{-\mathbf{1}}\right)$ \\
\hline 0.0 & 0.33 & 4.05 \\
\hline 2.5 & 0.28 & 1.57 \\
\hline 5.0 & 0.26 & 1.18 \\
\hline 7.5 & 0.22 & 0.29 \\
\hline 10 & 0.21 & 0.34 \\
\hline
\end{tabular}

Table (2): Conducting parameters for the system $\mathrm{Se}_{70} \mathrm{Te}_{30-\mathrm{x}} \mathrm{In}_{\mathrm{x}}$, film samples:

$$
\sigma_{00}=2.9 \times 10^{-6} \mathrm{~S} \mathrm{~cm}^{-1},\left(\mathrm{KT}_{\mathrm{MN}}\right)^{-1}=26.76(\mathrm{e} \mathrm{V})^{-1}
$$

\begin{tabular}{|c|c|c|}
\hline In Con. $(\boldsymbol{a t} \%)$ & $\begin{array}{c}\Delta \boldsymbol{E}(\boldsymbol{e} \boldsymbol{V}) \\
\mathbf{+ 0 . 0 3}\end{array}$ & $\begin{array}{c}\boldsymbol{\sigma}_{\boldsymbol{o}} \\
\left(\mathbf{S ~ c m}^{-\mathbf{1}}\right)\end{array}$ \\
\hline 0 & 0.24 & $1.92 \times 10^{-3}$ \\
\hline 2.5 & 0.77 & $5.94 \times 10^{3}$ \\
\hline 5 & 0.68 & $1.72 \times 10^{2}$ \\
\hline 7.5 & 0.63 & $2.15 \times 10$ \\
\hline 10 & 0.38 & $9.55 \times 10^{-2}$ \\
\hline
\end{tabular}




\section{Discussion:}

The increase of the dc conductivity with temperature, Figures $(1 \& 2)$, is believed to be due to an increase in the free carrier concentration (holes) due to the statistical shift of the Fermi level. Comparing Figures ( $1 \& 2)$ and Tables $(1 \& 2)$ one can say that conductivities of film samples are about three orders of magnitude lower than those of the bulk samples. The main reason is the difference in the electronic density of states for films (two-dimensional system) and that of bulk samples. The considerable decrease in conductivity on incorporation of In to the binary $\mathrm{Se}_{70} \mathrm{Te}_{30-\mathrm{x}}$ film could be due to a change in the cross-linking of Se-Te chains which enhances the disorder in the system in agreement with Dixit and Kumer [12]. The linear increase in $\sigma_{0}$ values with increasing $\Delta \mathrm{E}$, Figures $(2 \& 4)$, proved the validity of $\mathrm{MN}$ rule in the investigated samples.

In chalcogenides the interesting phenomena concerning the electrical conductivity near room temperature could be considered through two schools, the Mott-Davis [13] and the Emin one [14]. On the basis of Mott's model and at room temperature the carriers are excited across the mobility edge into extended states. Mott estimated a constant value of $\sigma_{0}$ in chalcogenides $=$ $100 \mathrm{Scm}^{-1}$. In Emin's model a small polaron formation is suggested. The electrical conductivity associated with around room temperature small polaron motion is essentially thermally activated; $\sigma_{0}$ will vary little between specimens and predicted to be $\left(10^{2}-10^{3} \mathrm{~S} \mathrm{~cm}^{-1}\right)$.

Considering the data in the present work it can be seen that $\sigma_{0}$ does not have a constant value as predicted by Mott. Rather a huge difference was observed in bulk and film samples. Even in the bulk specimens, the obtained values are very much lower than the $\sigma_{0}$ predicted by the model. Therefore the model can not be applied in such chalcogenides. Besides the observed difference in $\sigma_{0}$ values suggests that $\sigma_{0}$ can not be regarded as a microscopic conductivity. Instead $\sigma_{00}$ may have the physical meaning in terms of a microscopic conductivity. On the other hand the magnitude of predicted $\sigma_{0}$ for small polaron hopping is very much larger than the estimated values in bulk samples and most films. Also as shown in Table II $\sigma_{0}$ changes too much when indium concentration changes. Hence the polaron model is also unable to explain the dc transport in the system $\mathrm{Se}_{70} \mathrm{Te}_{30-\mathrm{x}} \operatorname{In}_{\mathrm{x}}$.

On the other side the $\mathrm{E}_{\mathrm{MN}}$ values estimated in the present study (4-21 meV) were found in a good agreement with the published data [15]. Besides very low values of $\sigma_{00}\left(10^{-5}-10^{-15} \mathrm{~S} \mathrm{~cm}^{-1}\right)$ for organic semiconductors were reported [16]. In such materials the electrical transport is assumed to be dominated by electron tunneling through intermolecular barriers which leads to 
small tunneling factor and thereby small $\sigma_{00}$. A similar situation may occur in some chalcogenides and thereby $\sigma_{00}$ becomes very small. However the high activation energy values and the big difference between $\sigma_{00}$ in the present work and those predicted by this model put restrictions on the conduction process in the present system to be due to tunneling.

How then can this discrepancy be explained?. The validity of the MN rule and the obtained activation energy values speculates that $S_{70} T_{30-x} I_{x}$ $(0<x<10)$ system gives a wide band gap semiconductor with exponential localized tail states as proposed by Roberts [10]. Accordingly the argument is that the major contribution of the conduction processes does not come from the region of Fermi level but more likely from these tail states which extend exponentially towards a finite energy value near the midgap. Hence $\sigma_{0}$ could not have the same value for all samples, since the energetic positions of these tail states are strongly influenced by addition of In content. The big difference in $\sigma_{0}$ values observed in the films indicated that $\sigma_{0}$ can not have the physical meaning in terms of a microscopic conductivity. In addition and instead of the tunneling model, a hopping model is proposed. In fact a hop over about $0.27 \mathrm{e}$ $\mathrm{V}$ and 0.5 e V obtained for bulk and film samples, respectively, needs more than one phonon suggesting a multiphonon effect to play the major role for transport in these compounds. The localized density of state distribution suggests the existence of different values for Meyer-Neldel energy. The values reported in the present study $(4-21 \mathrm{meV})$, depending on indium concentration, support this idea.

\section{Conclusions:}

The resistivity of film samples in the system $\mathrm{Se}_{70} \operatorname{Te}_{30-\mathrm{x}} \operatorname{In}_{\mathrm{x}}(0<\mathrm{x}<10)$ at. $\%$ were found three orders higher than the bulk ones. Addition of In to the binary chalcogenide $\mathrm{Se}_{70} \mathrm{Te}_{3-\mathrm{x}}$ led to a marked decrease in the conductivity. An experimental evidence for the correlation between $\sigma_{0}$ and $\Delta \mathrm{E}$ in both bulk and film samples was obtained. The estimated $\sigma_{00}\left(3.75 \times 10^{-3} \& 2.9 \times 10^{-6} \mathrm{~S} \mathrm{~cm}^{-1}\right.$ for bulk and film forms respectively) can be regarded as a microscopic conductivity, instead of $\sigma_{0}$.

\section{References:}

1. Shimakawa K and Abdel-Wahab, Appl.Phys.Lett., 70, 652 (1977).

2. Coutts T.J and Pearsall N.M, Appl. Phys. Lett., 44, 134 (1984).

3. Wanger D. Irsingler P and Dunstant D.J,J.Non.Cryst. Solids, 59\&60, 413 (1983).

4. Djamdji F and LeComber P.G, Phil. Mag., B 56,31 (1987).

5. Fortner J, Karpov V.G, and Saboung I, Appl. Phys. Lett., 66, 977(1994). 
6. Narasimhan K.L, and Arora B.M, Solid State Commum., 55, 615 (1985).

7. Kemeny G, and Rosenberg B, J.Chem. Phys., 52, 4151 (1970).

8. Irsingler P, Wanger D, and Dunstant D.J, Phys. C, 16, 6605. (1983).

9. Dwivedi S.K, Dixit M, Kumar A, J. of Mat. Sci. Lett., 17233 (1998).

10. Roberts G.G, J. Phys.C, 4, 3167 (1971).

11. Jackson W.B, Phys. Rev. B, 38, 3595 (1988).

12. Dixit M, Kumar A, Physica B, 252, 286-294 (1989).

13. Mott N.F, Davis E.A and Street R.A, Phil. Mag., 32,961 (1975).

14. Emin D, Seager C.H, Quinn R.K, Phys. Rev.Lett., 28, 813 (1972).

15. Abd el-Wahab F, Ph. D. Thesis, Gifu Univ., Japan (1998).

16. Rosenberg B, Bhowmik B.B, Harder H.C and Postrow J.Chem Phys. 49, 4108 (1968). 